\title{
RELAÇÃO ENTRE SÍNDROME DE BURNOUT, ANSIEDADE E QUALIDADE DE VIDA ENTRE ESTUDANTES DE CIÊNCIAS DA SAÚDE
}

\author{
Gustavo Magalhães VIANA ${ }^{1}$ \\ Thiago Gonçalves SILVA ${ }^{2}$ \\ Camillo Tupinambá OLIVEIRA ${ }^{3}$ \\ Matheus Felipe Rodrigues CASTRO ${ }^{4}$ \\ Danilo Lima CARREIRO 5 \\ Laura Tatiany Mineiro COUTINHO \\ Andréa Maria Eleutério de Barros Lima MARTINS ${ }^{7}$ \\ Wagner Luiz Mineiro COUTINHO ${ }^{8}$
}

\begin{abstract}
1. Estudante do Curso de Graduação em Medicina da Associação Educativa do Brasil (SOEBRAS), gustavomv89@gmail.com

2. Estudante do Curso de Graduação em Medicina da SOEBRAS, thiago.medicina@live.com

3. Estudante do Curso de Graduação em Medicina da SOEBRAS, camillotupinamba@gmail.com

4. Estudante do Curso de Graduação em Medicina da SOEBRAS, matheusfrc92@ gmail.com

5. Mestre em Ciências da Saúde pela Universidade Estadual de Montes Claros (Unimontes). Professor das Faculdades

Santo Agostinho (FASA) e da SOEBRAS, danilolimacarreiro@gmail.com

6. Mestre em Ciências da Saúde pela Unimontes. Professora da SOEBRAS, mineiro.laura@ @mail.com

7. Doutora em Saúde Pública pela Universidade Federal de Minas Gerais (UFMG). Professora do Programa de Pós

Graduação em Ciências da Saúde da Unimontes, martins.andreamebl@gmail.com

8. Mestre em Ciências da Saúde pela Unimontes. Professor da SOEBRAS, coutinhowlm@gmail.com
\end{abstract}

\section{Recebido em: 30/05/2014 - Aprovado em: 30/06/2014 - Disponibilizado em: 30/07/2014}

\begin{abstract}
RESUMO: Este estudo teve por objetivo determinar a prevalência da Síndrome de Burnout e dimensões (exaustão emocional, descrença, eficácia profissional) e avaliar sua relação com transtorno de ansiedade e com percepção do nível de qualidade de vida entre estudantes de Ciências da Saúde. Caracteriza-se como estudo transversal analítico, tendo sido utilizados para coleta de dados: Maslach Burnout Inventory Student Survey, Inventário de Ansiedade Traço-Estado, WHOQOL-Bref, Critério de Classificação Econômica Brasil e Questionário demográfico-socioeconômico, condições de saúde e discentes. Identificaram-se prevalências da Síndrome de Burnout em 65,1\% dos estudantes (n=229); alto nível de exaustão emocional em $35,2 \%$ ( $n=124)$; alto nível de descrença em $35,8 \%$ (n=126) e baixo nível eficácia profissional em 30,4\% ( $n=107)$. Registrou-se maior chance de desenvolver Síndrome de Burnout entre estudantes sem filhos, com altos índices de ansiedade-traço e com baixa percepção do nível de qualidade de vida no domínio físico. Maiores chances de desenvolver exaustão emocional foram evidentes entre aqueles com altos índices de ansiedade-estado e com baixa percepção do nível de qualidade de vida no domínio psicológico. Maior chance de desenvolver a dimensão eficácia profissional entre aqueles com altos índices de ansiedade-estado e com baixa percepção do nível de qualidade de vida no domínio social. Constatou-se relação entre SB e suas dimensões com transtorno de ansiedade e com percepção do nível de qualidade de vida, evidenciando a necessidade de se adotar medidas de enfrentamento da síndrome para possível redução do transtorno de ansiedade e promoção do nível de qualidade de vida entre estudantes de Ciências da Saúde.
\end{abstract}

Palavras-chave: Esgotamento Profissional. Ansiedade. Qualidade de vida. Estudantes de Ciências da Saúde.

\section{RELATIONSHIP BETWEEN BURNOUT SYNDROME, ANXIETY AND QUALITY OF LIFE AMONG HEALTH SCIENCES STUDENTS}

\begin{abstract}
The aim of this study was to determinate the prevalence of Burnout Syndrome and its dimensions (emotional exhaustion, professional efficacy and disbelief) and evaluate its relationship with anxiety disorders and quality of life levels among health science students. This is a analytic transversal study, which used Maslach Burnout Inventory Student Survey, Anxiety inventory, WHOQOL - Bref, Critério de Classificação Econômica Brasil and demographic questionnaires, health and students conditions. Burnout syndrome had prevalence of $65,1 \%$; high emotional exhausting in 35,2\% ( $\mathrm{n}=124)$; high disbelief rates in 35,8\% $(\mathrm{n}=126)$ and low professional efficacy levels in $30,4 \%(\mathrm{n}=107)$. Students without kids, with high trace-anxiety levels and low quality of live perception in physics domain, had registry bigger chance to develop Burnout syndrome. Higher chances to develop emotional exhaustion were evident between those who had high state-anxiety associated with low quality of life perception in psychological domain. Those with high state-anxiety level and low quality of life perception in social domain presented bigger chance
\end{abstract}


to develop professional efficacy. This study found a relationship between SB and its dimensions with anxiety disorders and quality of life levels. That result showed a necessity to develop ways to deal with this syndrome, so anxiety disorders could decrease and quality of life promotion among health science students increase.

Key Word: Professional exhaustion. Anxiety. Quality of life. Health Science Students.

\section{INTRODUÇÃO}

A Síndrome de Burnout (SB) decorre da exposição crônica a agentes estressores na qual a pessoa não dispõe de mecanismos de enfrentamento suficientes para confrontar tais agentes e assim acaba por desenvolver a síndrome constituída pelas dimensões: exaustão emocional, despersonalização e baixa realização profissional. (MASLACH e JACKSON, 1981; PEREIRA, 2010).

Apesar da classe estudantil não ser considerada como trabalhadora, as atividades estudantis podem ser tidas como préprofissionais por inserirem numa estrutura organizacional com obrigatoriedade de desenvolver atividades específicas como estudar e confrontar-se com aulas práticas, estágios e atividades avaliativas e pelo ambiente acadêmico competitivo, gerador de conflitos e de estresse. (BALOGUN et al., 1995; SCHAUFELI e TARIS, 2005; CARLOTTO e CÂMARA, 2006; CAMPOS e MAROCO, 2012; TOMASCHEWSKIBARLEM et al., 2012). Entre estudantes a SB se constitui pelas dimensões: exaustão emocional (EE), descrença (DE) e eficácia profissional (EP). (MARTÍNEZ, PINTO e SILVA, 2000).

Estudos têm sido realizados com o intuito de melhor compreender a SB, inclusive entre estudantes, todavia ainda são incipientes pesquisas que avaliaram sua relação com transtorno de ansiedade e com percepção do nível de qualidade de vida (QV). Pesquisas internacionais entre estudantes de medicina registraram aumento da frequência da SB e do transtorno de ansiedade ao longo do curso, (WILLCOCK et al., 2004), sendo que estudantes não acometidos por SB tendem a apresentar melhor percepção do nível de QV. (DYRBYE et al., 2010).

Com o intuito de melhor compreender as relações entre $\mathrm{SB}$, transtorno de ansiedade e percepção do nível de QV, este estudo propôs determinar a prevalência da $\mathrm{SB}$ e dimensões e avaliar tais relações entre estudantes de Ciências da Saúde.

\section{METODOLOGIA}

Estudo epidemiológico transversal entre estudantes dos cursos de graduação em Ciências da Saúde (Biomedicina, Educação Física, Enfermagem, Farmácia, Fisioterapia, Nutrição e Psicologia) de uma instituição de ensino superior de Montes Claros - MG. Procedeu-se à coleta dos dados por pesquisadores previamente treinados, considerando-se o período no qual os estudantes não realizassem atividades avaliativas. A princípio obteve-se junto à 
instituição de ensino o número de estudantes matriculados e cursando os referidos cursos, identificando-se uma população de 3861 pessoas. Realizou-se então cálculo amostral considerando nível de confiança de 95\%, margem de erro de $5 \%$ e prevalência de SB de $50 \%$ identificando-se a necessidade da participação de 350 estudantes que foram selecionados por amostragem aleatória probabilística estratificada, considerando proporcionalidade por turnos, cursos e períodos.

Para avaliar a SB empregou-se o questionário estruturado Maslach Burnout Inventory Student Survey (MBI-SS) validado para uso no Brasil (EE e EP respectivos alfas de Cronbach: 0,81 e 0,74; DE alfa de Cronbach: 0,59). (CARLOTTO e CÂMARA, 2006). Avalia-se Burnout de acordo com escores obtidos em cada dimensão, onde altos níveis em EE e DE e baixos níveis em EP indicam alto nível de SB. (CARLOTTO e CÂMARA, 2008). Para determinar EE e DE utilizou-se o percentil 66 e para EP o percentil 33. (MASLACH e JACKSON, 1986). A SB e suas dimensões foram consideradas variáveis dependentes. As variáveis independentes foram classificadas em individuais, condições de saúde e discentes. Na Tabela 1 são apresentadas as categorizações das variáveis em estudo.

A análise estatística se deu pelo software Statistical Package for the Social Sciences v.17.0. Para avaliar associação entre SB e dimensões às variáveis independentes, realizou-se análise bivariada através do Teste Qui-quadrado considerando associação ao nível de $p \leq 0,20$. Variáveis independentes que se mostraram associadas foram incluídas nas análises de regressão logística múltipla retendo-se no modelo final aquelas que se associaram com SB e dimensões ao nível de $\mathrm{p} \leq 0,05$.

Estudo aprovado pelo Comitê de Ética e Pesquisa da SOEBRAS com parecer consubstanciado 141.033 .

Tabela 1 - Categorizações das variáveis, Síndrome de Burnout entre estudantes do nível superior, Montes Claros - MG, $2013(n=352)$

\begin{tabular}{|c|c|c|}
\hline Variáveis & Categorização & Referência para categorização \\
\hline \multicolumn{3}{|l|}{ Dependentes } \\
\hline Síndrome de Burnout & $0=$ ausência $1=$ presença & Carlotto e Câmara (2006) \\
\hline Exaustão emocional & $0=$ ausência $1=$ presença & Carlotto e Câmara (2006) \\
\hline Descrença & $0=$ ausência $1=$ presença & Carlotto e Câmara (2006) \\
\hline Eficácia profissional & $0=$ ausência $1=$ presença & Carlotto e Câmara (2006) \\
\hline \multicolumn{3}{|l|}{ Independentes } \\
\hline \multicolumn{3}{|l|}{ Individuais } \\
\hline \multicolumn{3}{|l|}{ Demográficas } \\
\hline Sexo & $0=$ masculino $1=$ feminino & Carlotto (2011) \\
\hline Idade & $0=>22$ anos $1=\leq 22$ anos & Trindade e Lautert (2010) (mediana) \\
\hline Raça ou cor da pele ${ }^{a}$ & $0=$ branca/amarela $1=$ preta $/$ parda & - \\
\hline \multicolumn{3}{|l|}{ Socioeconômicas } \\
\hline Estado civil $^{\mathrm{a}, \mathrm{b}}$ & $0=$ casado $1=$ solteiro/divorciado/desquitado & Maslach e Leiter (1997) \\
\hline Filhos & $0=\operatorname{sim} 1=$ não & Carlotto (2011) \\
\hline Trabalha & $0=$ não $1=\operatorname{sim}$ & Maslach (1982) \\
\hline Segmentação econômica ${ }^{c}$ & $0=\operatorname{alta}(\mathrm{A} 1 / \mathrm{B} 2) 1=$ baixa $(\mathrm{C} 1 / \mathrm{E})$ & Rechenchosky et al. (2009) \\
\hline
\end{tabular}




\begin{tabular}{|c|c|c|}
\hline Uso de medicamentos (curso superior) & $0=$ não $1=\operatorname{sim}$ & - \\
\hline Tratamento psicoterápico atual & $0=$ não $1=\operatorname{sim}$ & - \\
\hline Percepção nível QV geral $^{\mathrm{d}}$ & $0=$ alta $1=$ baixa & Silva e Barreto (2012) (menor quartil) \\
\hline Percepção nível QV domínio físico ${ }^{\mathrm{d}}$ & $0=$ alta $1=$ baixa & Silva e Barreto (2012) (menor quartil) \\
\hline Percepção nível QV domínio psicológico ${ }^{\mathrm{d}}$ & $0=$ alta $1=$ baixa & Silva e Barreto (2012) (menor quartil) \\
\hline Percepção nível QV domínio social ${ }^{\mathrm{d}}$ & $0=$ alta $1=$ baixa & Silva e Barreto (2012) (menor quartil) \\
\hline Percepção nível QV domínio ambiental ${ }^{\mathrm{d}}$ & $0=$ alta $1=$ baixa & Silva e Barreto (2012) (menor quartil) \\
\hline Ansiedade-Traço ${ }^{\mathrm{e}}$ & $0=$ baixa $1=$ alta & mediana \\
\hline Ansiedade-Estado $^{\mathrm{e}}$ & $0=$ baixa $1=$ alta & mediana \\
\hline \multicolumn{3}{|l|}{ Condições discentes } \\
\hline Turno & $0=$ diurno e $1=$ noturno & - \\
\hline Períodos cursados & $0=$ iniciais e $1=$ finais & - \\
\hline \multicolumn{3}{|c|}{ 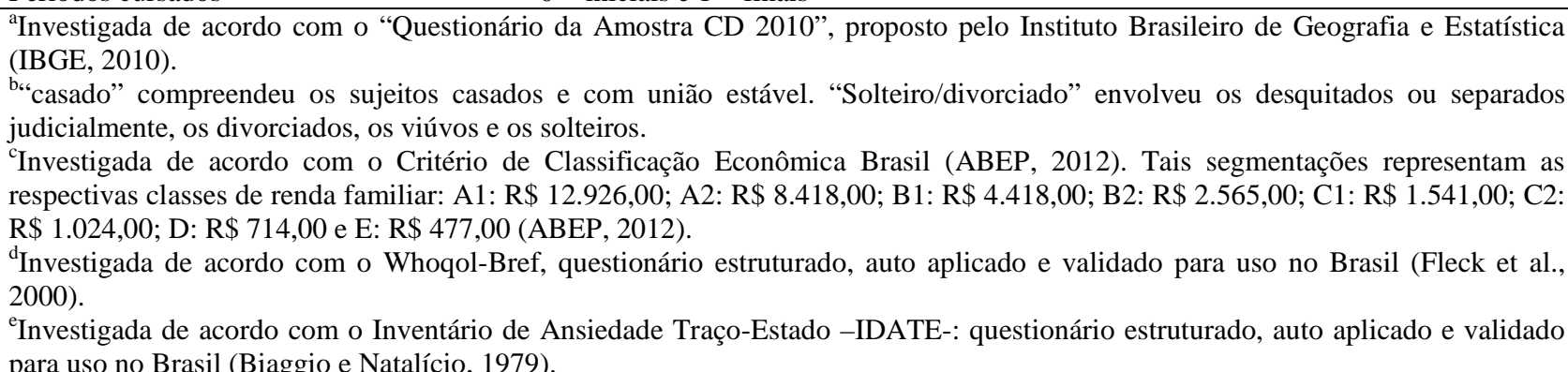 } \\
\hline
\end{tabular}

\section{RESULTADOS E DISCUSSÃO}

Participaram do estudo 352 estudantes com média de idade de 23,87 anos $( \pm 0,366$; $\mathrm{IC}_{95 \%}$ : 23,15-24,59; 18-63) registrando-se prevalências de SB em 65,1\% (n=229) dos estudantes; alto nível de EE em 35,2\% ( $\mathrm{n}=124) ;$ alto nível de DE em 35,8\% (n=126) e baixo nível EP em 30,4\% ( $n=107)$. Resultados divergentes de pesquisas brasileiras entre estudantes de Ciências da Saúde nas quais se identificaram prevalências da SB de 17,0\% (CAMPOS et al., 2012) e de 0\% (CARLOTTO, NAKAMURA e CÂMARA; 2006); alto nível de EE de 54,0\%; alto nível de DE em $24,0 \%$ e baixo nível de EP de 46,0\%. (VASCONCELOS et al., 2012).

Apesar da divergência de resultados, há de se considerar as expressivas prevalências registradas tanto no presente estudo quanto nas pesquisas prévias, uma vez que o desenvolvimento da SB durante o período acadêmico pode implicar em possíveis dificuldades tanto acadêmicas como profissionais (MARTÍNEZ et al., 2002) sobretudo ao se considerar estudantes de Ciências da Saúde, que por prestarem cuidados diretos a outras pessoas mostram-se ainda mais susceptíveis aos agentes estressores. (GIL-MONTE, 2002). Neste sentido, a identificação do desenvolvimento da SB, ainda durante o período acadêmico, pode propiciar a elaboração de estratégias de enfrentamento de agentes estressores, com consequente prevenção da síndrome e benefícios para estudantes, educadores, futuros profissionais, empregadores e pacientes. (BALOGUN et al., 1995; MARTÍNEZ et al., 2002).

Na Tabela 2 são apresentados os resultados do modelo de regressão logística múltipla. Registrou-se maior chance de desenvolver SB entre estudantes com altos índices de ansiedade-traço, com baixa 
percepção do nível de qualidade de vida no domínio físico e entre aqueles sem filhos.

Não se identificaram pesquisas nacionais acerca da relação entre $\mathrm{SB}$, dimensões e transtorno de ansiedade entre estudantes. Estudos internacionais com estudantes de medicina identificaram aumento das prevalências da SB e do transtorno de ansiedade ao longo do curso; (WILLCOCK et al., 2004); entre estudantes acometidos por estresse também foram identificados indícios de transtorno de ansiedade; (PÖHLMANN et al., 2005); tendo sido registrada associação entre percepção negativa do nível de QV e SB (DYRBYE, 2010).

Tabela 2 - Modelos múltiplos de regressão logística das variáveis preditoras da SB e dimensões, estimativa da OR e IC 95\%, estudantes do ensino superior, Montes Claros - MG, 2013 ( $\mathrm{n}=352$ )

\begin{tabular}{|c|c|c|c|c|c|c|c|c|c|}
\hline \multirow{2}{*}{$\begin{array}{l}\text { VARIÁVEIS } \\
\text { INDIVIDUAIS }\end{array}$} & \multicolumn{3}{|c|}{ SB } & \multicolumn{3}{|c|}{ EE } & \multicolumn{3}{|c|}{ EP } \\
\hline & & & & & & & & & \\
\hline Socioeconômicas & OR & $\mathrm{IC}_{\mathbf{9 5} \%}$ & $p$ & OR & $\mathrm{IC}_{\mathbf{9 5} \%}$ & $p$ & OR & $\mathbf{I C}_{\mathbf{9 5} \%}$ & $p$ \\
\hline Filhos & & & & & & & & & \\
\hline Sim & 1,00 & & & - & & & - & & \\
\hline Não & 2,00 & $1,07-3,76$ & 0,030 & - & - & - & - & - & - \\
\hline Condições de saúd & & & & & & & & & \\
\hline Percepção QV dor & & & & & & & & & \\
\hline Alta & 1,00 & & & - & & & & - & - \\
\hline Baixa & 3,58 & $1,65-7,79$ & 0,001 & - & - & - & - & - & - \\
\hline Percepção QV dor & & & & & & & & & \\
\hline Alta & - & & & 1,00 & & & - & & \\
\hline Baixa & - & - & - & 2,46 & $1,47-4,14$ & 0,001 & - & - & - \\
\hline Percepção QV dor & & & & & & & & & \\
\hline Alta & - & & & - & & & 1,00 & & \\
\hline Baixa & - & - & - & - & - & - & 1,82 & $1,00-3,33$ & 0,05 \\
\hline Ansiedade-Traço & & & & & & & & & \\
\hline Baixa & 1,00 & & & - & & & - & & \\
\hline Alta & 2,91 & $1,72-4,94$ & 0,000 & - & - & - & - & - & - \\
\hline Ansiedade-Estado & & & & & & & & & \\
\hline Baixa & - & & & 1,00 & & & 1,00 & & \\
\hline Alta & - & - & - & 2,86 & $1,47-4,14$ & 0,000 & 2,18 & $1,33-3,57$ & 0,002 \\
\hline
\end{tabular}

A associação identificada entre SB e baixa percepção do nível de QV no domínio físico pode ser explicada pelo fato de tal domínio compreender aspectos como energia e fadiga, dor e desconforto, sono e repouso. (TABELEÃO et al., 2011). Nesse sentido, Burnout foi primeiramente descrito na década de 1970 como sentimento de fracasso e exaustão causado por excessivo desgaste energético e de recursos. Dentre as consequências que pode acarretar se encontram quadros álgicos como artromialgias, cefaleias e enxaquecas, (CHERNISS, 1980), sendo consideradas como estratégias de enfrentamento, inclusive durante o período acadêmico, os cuidados com o sono, a organização do tempo e o equilíbrio entre estudo e lazer. (ZONTA et al., 2006). Desta forma, tal associação pode fundamentar-se nas peculiaridades impostas 
aos estudantes, principalmente aos de Ciências da Saúde, como inserção em ambiente competitivo entre pares, professores e supervisores; e indisponibilidade temporal para atividades sociais (BALOGUN et al., 1995; ENNS et al., 2001) favoráveis ao desgaste energético, à fadiga, aos quadros álgicos e ao distúrbio de sono.

A menor chance de ocorrer SB entre estudantes que declararam "ter filhos" pode indicar uma fonte de gratificação no fato do estudante cuidar do filho, além de uma melhor delimitação temporal e distanciamento dos agentes estressores acadêmicos. (CARLOTTO, NAKAMURA e CÂMARA; 2006). Estudo prévio entre professores brasileiros identificou associação entre as dimensões da SB e o fato do professor não se ter filhos. (CARLOTTO, 2011).

Maiores chances de desenvolver EE foram evidentes entre aqueles com altos índices de ansiedade-estado e com baixa percepção do nível de qualidade de vida no domínio psicológico. A dimensão $\mathrm{EE}$ relaciona-se ao desgaste em relação aos estudos e às atividades acadêmicas. (CARLOTTO e CÂMARA, 2006). Por sua vez, a ansiedade-estado apresenta-se como um estado emocional transitório pertinente a um momento ou situação específica. (FERREIRA et al., 2009). Neste sentido tal associação pode fundamentar-se na expressiva carga de estresse à qual os estudantes são expostos devido aos longos períodos de estudos; às cobranças pessoais por parte de docentes e familiares; bem como ao estado maturacional transitório entre a adolescência e a idade adulta que pode levar o estudante a experimentar um período de crise diante o novo papel social a ser assumido, o de jovem adulto. (CAPLAN, 1980; SILVER, 1982; PAPALIA e OLDS 2000).

O domínio psicológico da percepção do nível de QV envolve sentimentos positivos, pensamento, aprendizagem, memória e concentração, autoestima, imagem corporal, aparência e sentimentos negativos. (TABELEÃO et al., 2011). A maior chance de desenvolver EE entre estudantes com baixa percepção neste domínio pode basear-se nas particularidades que envolvem os cursos de graduação em Ciências da Saúde como: contato com pessoas acometidas por doenças graves, dor, sofrimento e com a própria morte, que pode gerar no estudante conflito entre o racional e o emocional; cotidianos marcados por sentimentos de dúvidas, incertezas, expectativas, ansiedade, medo, tristeza, raiva, angustia, decepção e impotência relacionados principalmente às primeiras práticas terapêuticas; insuficiente preparo psicológico destes para enfrentar tais situações; pressão em relação ao processo de aprendizagem; expressivo fluxo de novas informações; e vivência constante de novas experiências em salas de aula, laboratórios e na assistência desenvolvida nos campos de estágios, (ENNS et al., 2001; NOGUEIRA- 
MARTINS, 2002; BORGES e CARLOTTO, 2004), fatos estes favoráveis ao desenvolvimento de sentimentos de exaustão e aos sentimentos envolvidos no domínio psicológico da percepção do nível de QV.

Maiores chances de desenvolver EP foram evidentes entre aqueles com altos índices de ansiedade-estado e com baixa percepção do nível de qualidade de vida no domínio social. A dimensão EP é demarcada por um sentimento de incompetência enquanto estudante. (CARLOTTO e CÂMARA, 2006). A associação entre EP e altos índices de ansiedade-estado pode justificar-se pelo estado emocional pertinente a momentos ou situações específicas como constantes atividades avaliativas; limitações de conhecimento científico e possibilidades de atuação, com consequente receio do estudante em cometer erros prejudiciais aos pacientes e ao reconhecimento por pares, professores e supervisores (NOGUEIRAMARTINS, 2002; PÖHLMANN et al., 2005).

O domínio social da percepção do nível de QV engloba relações pessoais; suporte e apoio social; e atividade sexual (TABELEÃO et al., 2011). A maior chance de desenvolver EP entre estudantes com baixa percepção neste domínio pode fundamentar-se nas peculiaridades das relações sociais acadêmicas que podem ser delimitadas por competitividade, por constante busca pelo perfeccionismo e por incertezas, ansiedades, expectativas, receios, sentimentos de decepção e impotência que demarcam principalmente os primeiros momentos do relacionamento estudante-paciente. (HENNING et al., 1998; NOGUEIRAMARTINS, 2002). A este processo soma-se ainda as possíveis dificuldades do estudante em trocar informações e conhecimentos com pares, professores e supervisores levando-o a ocultar ansiedades e incertezas através de atitudes defensivas que pode culminar numa permanente dificuldade de relacionar-se com os pacientes. (TAHKA, 1988). Ressalta-se que entre profissionais de saúde, o intenso contato profissionais-pacientes-familiares apresenta-se como um dos fatores preditivos para a SB, uma vez que tal relação geralmente permeia sentimentos de ansiedade, tensão, medo e hostilidade encoberta, (RODRIGUEZ-MARÍN, 1995), sendo que tais experiências podem ser vivenciadas pelo estudante de Ciências da Saúde ainda no período de formação acadêmica, diante a realização das atividades práticas de estágios.

Salienta-se que o processo que relaciona a avaliação da SB e variáveis investigadas é dinâmico. Portanto, causas e efeitos certamente variam ao longo do tempo e, sendo este um estudo seccional, não é possível estabelecer uma relação temporal entre as associações observadas. Todavia, destaca-se que os resultados apresentados são confiáveis, frutos de um estudo epidemiológico. A principal contribuição desta pesquisa aos desenvolvidos previamente 
foi o desenho metodológico que propôs avaliar associações ainda não analisadas entre estudantes, independente do nível de ensino, motivando novas discussões sobre o tema.

\section{CONCLUSÃO}

A prevalência da $\mathrm{SB}$, considerada nesse estudo como altos níveis em EE e DE e baixos níveis em EP é preocupante. No modelo final ajustado registrou-se maior chance de desenvolvimento da SB entre estudantes com altos índices de ansiedadetraço quando comparados aos com baixos índices; entre os com baixa percepção do nível de qualidade de vida no domínio físico quando comparados àqueles com alta percepção; e entre os que relataram não ter filhos quando comparados aos que relataram os ter. Maior chance de desenvolver a dimensão EE foi identificada entre os estudantes com altos índices de ansiedadeestado quando comparados aos com baixos índices e entre os com baixa percepção do nível de qualidade de vida no domínio psicológico quando comparados àqueles com alta percepção. Registrou-se maior chance de desenvolver a dimensão EP entre estudantes com altos índices de ansiedade-estado quando comparados aos com baixos índices e entre os com baixa percepção do nível de qualidade de vida no domínio social quando comparados àqueles com alta percepção.

\section{REFERÊNCIAS}

ABEP. Associação Brasileira de Empresas de Pesquisa. Critério de Classificação

Econômica Brasil. São Paulo; 2012.

Disponível em <http://www.abep.org/new/> Acesso em 30 de maio de 2014.

BALOGUN, J. et al. Test-retest reability of a psychometric instrument designed to measure physical therapy student's Burnout. Perceptual and Motor Skill, Missoula, v. 81, p. 667-672, 1995.

BIAGGIO, A. M. B. NATALÍCIO, L. Manual para o Inventário de Ansiedade Traço Estado (IDATE). Rio de Janeiro: CEPA; 1979.

BORGES, A. M. B. CARLOTTO, S. Síndrome de Burnout e Fatores de Estresse em Estudantes de um Curso Técnico de Enfermagem. Aletheia, Canoas, v. 19, p. 4556, 2004.

CAMPOS, J. A. D. B. MAROCO, J. Adaptação transcultural Portugal-Brasil do Inventário de Burnout de Maslach para estudantes. Revista de Saúde Pública, São Paulo, v. 46, n. 5, p. 816-824, 2012.

CAMPOS, J. A. D. B. et al. Síndrome de Burnout em graduandos de Odontologia.

Revista Brasileira de Epidemiologia, São Paulo, v. 15, n. 1, p. 155-165, 2012.

CAPLAN, G. Princípios de psiquiatria preventiva. Rio de Janeiro: Zahar Editores; 1980.

CARLOTTO, M. S. CÂMARA, S.G. Características psicométricas do Maslach Burnout Inventory Student Survey (MBI-SS) em estudantes universitários brasileiros. Psico-USF, Itatiba, v. 11, n. 2, p. 167-173, 2006.

CARLOTTO, M.S. CÂMARA, S.G. Preditores da Síndrome de Burnout em estudantes universitários. Pensamiento 
Psicológico, Cali, v. 4, n. 10, p. 101-109, 2008.

CARLOTTO, M. S. NAKAMURA, A.P. CÂMARA, S.G. Síndrome de Burnout em estudantes universitários da área da saúde. PSICO, Porto Alegre, v. 37, n. 1, p. 57-62, 2006.

CARLOTTO, MS. Síndrome de Burnout em Professores: Prevalência e fatores associados. Psicologia: Teoria e Pesquisa, Brasília, v. 27, n. 4, p. 403-410, 2011.

CHERNISS, C. Staff burnout: job stress in the human service. Sage: Beverly Hills; 1980.

DYRBYE, L. N. et al. Factors associated with resilience to and recovery from burnout: a prospective, multi-institutional study of US medical students. Medical Education, Ottawa, v. 44, p. 1016-1026, 2010.

ENNS, M. W. et al. Adaptive and maladaptive perfectionism in medical students: a longitudinal investigation. Medical Education, Ottawa, v. 35, n. 11, p. 1034-1042, 2001.

FERREIRA, C. L. et al. Universidade, contexto ansiogênico? Avaliação de traço e estado de ansiedade em estudantes do ciclo básico. Ciência \& Saúde Coletiva, Rio de Janeiro, v. 14, n. 3, p. 973-981, 2009.

FLECK, MPA et al . Aplicação da versão em português do instrumento abreviado de avaliação da qualidade de vida "WHOQOLbref". Revista de Saúde Pública, São Paulo, v. 34, n. 2, p. 178-183, 2000.

GIL-MONTE, P. R. Influencia del género sobre el proceso de desarrollo del síndrome de quemarse por el trabajo (Burnout), em profesionales de enfermería. Psicologia em Estudo, Maringá, v. 7, n. 1, p. 3-10, 2002.

HENNING K, E. Y. S SHAW, D. Perfectionism, the impostor phenomenon and psychological adjustment in medical, dental, nursing and pharmacy students.

Medical Education, Ottawa, v. 32, v. 5, p. 456-464, 1998.

Instituto Brasileiro de Geografia e Estatística. CD 2010 - Questionário da Amostra. Censo 2010. Disponível em: $<$ http://www.censo2010ibge.gov.br/downloa d/questionarios/censo2010_amostra.pdf> Acesso em 30 de maio de 2014.

MARTÍNEZ, I. M. M. PINTO, A. M. SILVA, A.L. Burnout em estudantes do ensino superior. Revista Portuguesa de Psicologia, Lisboa, v. 35, p. 151-167, 2000.

MARTÍNEZ, I. M. M. et al. Burnout en estudiantes universitarios de España y Portugal. Ansiedad y estrés, Madrid, v. 8, n. 1, p. 13-23, 2002.

MASLACH, C. Burnout: The cost of caring. Englewood Cliffs. NJ: Prentice Hall; 1982.

MASLACH, C. JACKSON, S. E. The measurement of experienced burnout. J Occup Behav, v. 2, n. 2, p. 99-113, 1981.

MASLACH, C. JACKSON, S. E. Maslach Burnout Inventory manual. Palo Alto, University of California: Consulting Psychologist Press; 1986.

MASLACH, C. LEITER, M. P. The truth about burnout. How organizations cause personal stress and what to do about it. San Francisco: Jossey-Bass Inc; 1997.

NOGUEIRA-MARTINS, M. C. F. Humanização das relações assistenciais: a formação do profissional de saúde. São Paulo: Casa do Psicólogo; 2002.

PAPALIA, D. E. OLDS, S. W. Desenvolvimento humano. Porto Alegre: Artes Médicas Sul; 2000.

PEREIRA, A. M. T. B. Burnout: quando o trabalho ameaça o bem-estar do 
trabalhador. $4^{\mathrm{a}}$ ed. São Paulo: Casa do Psicólogo; 2010.

PÖHLMANN, K. et al. Stress, burnoutand health in the clinical period of dental education. European Journal of Dental Education, Berlim, v. 9, n. 2, p. 78-84, 2005.

RECHENCHOSKY, L. et al. Estado nutricional e perfil lipídico de crianças. Revista da Educação Física/UEM, Maringá, v. 20, p. 431-440, 2009.

RODRIGUEZ-MARÍN, J. Psicologia Social de la Salud. Madrid: Sínteses; 1995.

SCHAUFELI, W. B. TARIS, T. W. The conceptualization and measurement of burnout: Common ground and worlds apart. Work Stress, v. 19, n. 3, p. 256-62, 2005.

SILVA, L. S. BARRETO, S. M. Adverse psychosocial working conditions and poor quality of life among financial servisse employees in Brazil. Journal of

Occupational Health, v. 54, p. 88-95, 2012.

SILVER, H. K. Medical student and medical school. Jamaica, v. 247, p. 304-320, 1982.

TABELEÃO, V. P. et al. Qualidade de vida e esgotamento profissional entre docentes da rede pública de Ensino Médio e

Fundamental no Sul do Brasil. Caderno de

Saúde Pública, Rio de Janeiro, v. 27, n. 12, p. 2401-2408, 2011.

TAHKA V. O relacionamento médico paciente. Porto Alegre: Artes Médicas; 1988.

TOMASCHEWSKI-BARLEM, J. G. et al. Opção e evasão de um curso de graduação em enfermagem: percepção de estudantes evadidos. Revista Gaúcha de Enfermagem, Porto Alegre, v. 3, n. 2, p. 132-138, 2012.
TRINDADE, L. L. LAUTERT, L. Síndrome de Burnout entre os trabalhadores da Estratégia de Saúde da Família. Revista da Escola de Enfermagem da USP, São Paulo, v. 44, n. 2 p. 274-279, 2010.

VASCONCELOS. R.P. et al. A ocorrência da Síndrome de Burnout nos acadêmicos do último ano do curso de Fisioterapia. Revista Fisioterapia e Saúde Funcional, Fortaleza, v. 1, n. 1, p. 42-46, 2012.

WILLCOCK, S. M. et al. Burnout and psychiatric morbidity in new medical graduates. Medical Journal of Australia, Sydney, v. 181, p. 357-360, 2004.

ZONTA, R. ROBLES, A. C. C.

GROSSEMAN, S. Estratégias de enfrentamento do estresse desenvolvidas por estudantes de medicina. Revista Brasileira de Educação Médica, Rio de Janeiro, v. 30, n. 3, p. 147-153, 2006 